\title{
Pavie, une université au service des rois d'Espagne
}

\section{Pawia - uniwersyteł w służbie królów Hiszpanii}

\begin{abstract}
The era of Spanish domination over the University of Pavia, which lasted between the magnificent period of the Visconti and the Sforza reigns and the times of the reforms of Maria Teresa and Joseph II of Habsburg, is considered by some researchers as a period of decadence and by others as a phase of purely formal continuity.

Over the course of several centuries, there has been a transition from the idea of monolithic medieval university to flexible centers of higher education. From the sixteenth to the eighteenth centuries, the cycle of education at the secondary level was defined, which inevitably imposed its primacy over primary and higher education. In Pavia, the situation was no different from that of other European cities; on the contrary, it adopted certain values within the cultural policy carried out by the Spanish kings.

The aim of this article is - using the example of the University of Pavia - to present the changes that took place during this period in secondary education and at universities. The humanistic heritage of the University of Pavia during the Spanish period was confronted by the regional education system; it had to overcome the periods of crisis, then to face changes needed and to create the premises for a deliberate deployment of higher education, which would flourish later under the influence of the Enlightenment.
\end{abstract}

KEYWORDS

Pavia, university, education, Lombardy, training

\section{SLOWA KLUCZOWE}

Pawia, uniwersytet, edukacja, kształcenie, Lombardia

SPI Vol. 23, 2020/3

ISSN 2450-5358

e-ISSN 2450-5366 DOI: 10.12775/SPI.2020.3.009

Submitted: 02.09.2020 Accepted: 04.10.2020

Miscellanea 
Source research allowed for the formulation of conclusions that the Studium Ticinensis can be considered as an institution that was developing efficiently and was characterized by a coherent educational system, which - like in other regions of Europe - appears to be a fruit of modernity.

\section{ABSTRAKT}

Era hiszpańskiej dominacji nad Uniwersytetem w Pawii, która trwała między wspaniałym okresem panowania Viscontich i Sforzów, a czasami reform Marii Teresy i Józefa II Habsburga, uważana jest przez niektórych badaczy za okres dekadencji, a przez innych za etap formalnej ciqgłości w dziejach uczelni.

Na przestrzeni kilku stuleci nastapiło przejście od idei monolitycznego średniowiecznego uniwersytetu do elastycznych ośrodków kształcenia na poziomie wyższym. Od XVI do XVIII wieku określono cykl kształcenia na poziomie średnim, co nieuchronnie narzuciło jego prymat nad szkolnictwem podstawowym i wyższym. W Pawii sytuacja nie różniła się od tej w innych miastach europejskich, wręcz przeciwnie, przyięła ona pewne wartości w ramach polityki kulturalnej hiszpańskich królów.

Celem artykułu jest - na przykładzie Uniwersytetu w Pawii - przedstawienie zmian, jakie zaszły w tym okresie w szkolnictwie średnim i wyższym. Dziedzictwo humanistyczne Uniwersytetu w Pawii zostało skonfrontowane $w$ okresie hiszpańskim $z$ regionalnym systemem edukacji, musiało przezwyciężyć okresy kryzysu, a następnie stawić czoła potrzebnym zmianom i stworzyć przesłanki do świadomego wprowadzenia szkolnictwa wyższego, kłóre rozkwitnie później pod wpływem oświecenia.

Badania źródłowe pozwoliły na sformułowanie wniosków, że Studium Ticinensis można uznać za instytucję sprawnie się rozwijajacq, charakteryzująca się spójnym systemem edukacyjnym, który - podobnie jak w innych regionach Europy - jawi się jako owoc nowoczesności.

Positionnée entre l'éblouissante époque des origines sous les familles Visconti et Sforza et l'époque des réformes de Marie-Thérèse et Joseph II d'Habsbourg, l'ère de domination espagnole sur l'université de Pavie a été considérée, par certains, comme une période de décadence et, par d'autres, comme une phase de continuité purement 
formelle ${ }^{1}$. Il est évident que le nouvel élan accordé aux études relatives à cette période, grâce aussi à une meilleure connaissance des sources, a permis de considérer le Studium Ticinensis comme étant une institution en pleine évolution, tasseau d'un système éducatif articulé qui, comme dans d'autres régions d'Europe, se profile comme étant le fruit de la modernité. De l'université médiévale monolithique on est passé à des centres de formation de niveau supérieur flexibles, provoquant ainsi une situation apparemment ingérable mais qui, en même temps, a représenté une expérience importante et a marqué les prémisses de la nouvelle organisation autrichienne. $\mathrm{Du} \mathrm{XVI}^{\mathrm{e}}$ au XVIII ${ }^{\mathrm{e}}$ siècle, on définit le cycle scolaire secondaire qui, forcément, impose sa suprématie sur l'école primaire et sur l'enseignement universitaire. A Pavie, la situation n'est pas différente de celle existant dans d'autres sièges européens mais celle-ci assume au contraire des valeurs particulières dans le cadre de la politique culturelle des Rois d'Espagne. L'héritage humaniste de l'université de Pavie, durant la période espagnole, sera donc confronté à un système éducatif à vocation régionale surmontant les périodes de crise, connaissant des transformations critiquées et créant les prémisses d'une répartition réfléchie de l'enseignement supérieur qui s'épanouira sous l'influence des Lumières.

\section{L'héritage humaniste}

Dans les universités italiennes du XVe siècle, l'esprit humaniste avait alimenté une notion globale de renaissance qui, en se diffusant rapidement, avait déterminé la prise de conscience du retour nécessaire à l'art et à la pensée classiques.

La reconquête du savoir à travers les œuvres littéraires des antiques et le désir d'en assimiler l'état d'esprit se traduisaient dans l'aspiration à un nouveau modèle de vie orienté et issu, au point de vue éthique, de l'humanisme. Préparé par le travail des bibliophiles qui avaient, au cours des siècles précédents, par un long et patient travail de recherche, retrouvé les écrits fondamentaux de la culture

1 Pour tous approfondissements, voir les contributions à l'ouvrage collectif : Almum Studium Papiense. (2013). Storia dell'Università di Pavia. Volume 1: Dalle origini all'età spagnola, Tomo II: L'età spagnola, éd. D. Mantovani, Milano: Cisalpino. 
latine, l'humanisme italien se focalisait dans l'excessive individualité de Pétrarque chez qui les identités du mouvement se traduisaient en réalités fécondes.

Grâce à la longue amitié du poète avec Galéas II Visconti, Pétrarque séjourna pendant deux ans à Pavie, influençant, même si de façon indirecte, l'université de Pavie selon deux directions : le développement de la bibliothèque privée des Ducs ; l'orientation dans le choix des maitres de logique et de lettres. La domination des littéraires continua au XVe siècle avec l'engagement, au sein de l'université, de Antonio Beccadelli dit le Palermitain (en latin Antonius Panormita), de Laurent Valla et de Gasparin de Bergame (le Barzizza), lecteur de classiques latins, et enfin de François Philelphe, déjà élève de Barzizza à Padoue, qui arriva à Pavie en 1439 et qui, par ses travaux, marqua profondément la culture lombarde. Les humanistes se sentaient intégrés et étaient présents même à la cour de Ludovic Marie Sforza dit le More : parmi eux se distinguait George Merula, élève de Philelphe qui, pendant trois ans, commenta Cicéron à partir de la chaire de Pavie et qui ensuite, en passant sous la dépendance directe du Duc, composa l'Historia Vicecomitum, restée inachevée après sa mort. Le lien qui existait entre la cour des Visconti et des Sforza et l'université de Pavie plaçait cette dernière au centre des stratégies politico-culturelles du Duché, garantissant ainsi un rapport direct entre les institutions.

L'écho du prestige culturel pavesan, qui laissait présager les évènements qui allaient se passer peu de temps après, rejaillissait même sur la lointaine Castille, au point que des intellectuels du niveau de Gonzalo de Ayora, prestigieux représentant de la république des lettres et détenteur de la charge de Chroniqueur de la Couronne, choisirent justement Pavie comme siège pour l'acquisition d'une formation humaniste.

L'université de Pavie entrait dans l'ère moderne, forte de sa tradition et de sa renommée dans le domaine de l'enseignement juridique et valorisée dans le domaine littéraire par la présence des humanistes les plus connus et d'un groupe important d'étudiants provenant des territoires germaniques. Cet élan projettera graduellement le Studium au maximum de sa splendeur, position qu'il conservera pendant une grande partie du XVIe siècle et comptera un nombre important d'étudiants. 
Malgré les malaises politiques, à l'époque des guerres d'Italie, l'université continua à fonctionner parce que les gouvernements qui s'alternèrent, se proclamèrent toujours comme étant ses protecteurs. Les gouverneurs du Duché suivirent toujours de façon active la façon d'opérer de leur centre d'études majeur : François I, Roi de France, fut le rédacteur d'une lettre adressée au Podestà de Pavie " pro publicatione Edicti aperitionis Studii ad Festum S. Lucae » (Statuti e Ordinamenti 1925); et tenu informé du fait que les étudiants pavesans continuaient à être turbulents, il fit paraitre un décret le 5 juin 1520 " pro morigeratione studentium, ne incedant armati, sed talari veste indulti et se contineant " (Statuti e Ordinamenti 1925: 151).

En 1522, François II Sforza confirma, de nouveau par le décret du 5 juin, les immunités et les privilèges pour les maîtres et les élèves, défendant ainsi aux étudiants de l'État de s'inscrire dans une autre université que celle de Pavie, et en déclarant que « hora que la citade de Pavie à reducta fora de li strepiti bellici, dare opera chel Studio dessa citade sia florido et s'habia ad frequentare tanto per conservare la sua consueta et glorioa fama, quanto par adjutare li soi subditi ad seguire la virtute et studii litterarii in omne facultate " (Memorie e documenti 1877-78: 16-17). L'intention du Duc était celle de redonner un certain prestige à l'institution en s'engageant « a disponere le lecture in homini esperti et scientifici [...] in l'una et l'altra facultate cossi de Iuristi come de Artisti et studii d'humanità » (Memorie e documenti 1877-78: 17). Mais l'arrogance des étudiants ne diminua pas et l'on fut obligé de menacer de sanctions et de punitions quiconque aurait offensé le Recteur ou tout autre autorité académique.

Juste à la fin de la guerre franco-habsbourgeoise, allait commencer pour l'université une période bénéfique due à la présence de maîtres illustres et pendant laquelle celle-ci allait consolider ses structures logistiques, en se dotant d'équipements et d'institutions nouvelles. A Pavie, il n'existait pas un palais universitaire autonome et les salles de cours étaient installées dans les couvents ou dans d'autres bâtiments privés, tandis que les lecteurs les plus connus, suivis par un grand nombre d'élèves, donnaient leurs cours dans les églises. Progressivement, s'imposa l'idée de l'utilité de réunir toutes les facultés dans un unique bâtiment situé au centre de la ville. La première construction fut limitée à la partie septentrionale de l'édifice actuel, complétée et agrandie sous la domination autrichienne. Dans sa 
forme primitive, la construction avait une façade simple, avec deux portes et de larges gouttières, seize fenêtres à létage inférieur et dixhuit à l'étage supérieur, des salles intérieures imposantes avec un portail élégant à l'étage inférieur et une loggia à l'étage supérieur. L'initiative fut prise par le Sénat sur base d'un décret du 13 octobre 1533 et la construction fut commencée l'année suivante. Celle-ci fut érigée sur l'aire d'une maison appartenant à l'évêque de Pavie Azzon Visconti, fils de Galéas Ier et de Béatrice d'Este. L'approfondissement de la connaissance s'accompagnait d'une répartition logistique adéquate des laboratoires et des cabinets scientifiques à côté des salles de cours. La nouvelle construction était adjacente aux bâtiments de l'hôpital Saint-Mathieu, doté d'un fonds important qui, au fil du temps, avait augmenté considérablement grâce aux donations des bienfaiteurs.

\section{Le Studium espagnol}

La période pendant laquelle la Lombardie fut sous la domination espagnole détermina inévitablement toute une série de changements qui pesèrent sur l'organisation universitaire: même si la disposition de l'enseignement public continuait à être formellement réglée par les traditions ducales, dans la pratique ce type d'enseignement se distinguait de façon perceptible du modèle original malgré l'apparente solidité de la forme médiévale et malgré la politique de conservation explicitement déclarée par les autorités dirigeantes.

Les règles et les instruments qui, depuis la période des Sforza, garantissaient l'organisation des études, restèrent pratiquement les mêmes jusqu'en 1541, année durant laquelle le gouvernement de Charles Quint d'Habsbourg fit paraitre de nouvelles lois, le Constitutiones Domini Mediolanensis, d'où on apprend que l'université pavesane était dirigée par le Sénat de Milan, le plus haut organisme d'administration et de juridiction de l'Etat. Dans le premier livre des Constitutions, on lit en effet : "Universitatis quoque Papiensis curam habebit, Lectores deputabit, et amovebit, salaria constituet, et denique alia faciet, veluti facere consueverat » (Statuti e Ordinamenti 1925: 155 et suivants). Un siècle et demi après sa fondation, l'université était donc dépendante d'un organisme électif de l'État pour tout ce qui concernait l'administration et l'organisation académique, de la nomination et du licenciement des professeurs à l'attribution 
des prébendes. Un choix qui, d'un côté, était dicté par la volonté de garantir l'enracinement de l'université dans le territoire, sensible aux ingérences du patriciat local et qui, d'un autre côté, faisait que l'enseignement Pavesane était décentralisé par rapport au contrôle rigoureux exercé par le souverain sur les universités ibériques et, en partie aussi, sur celles de la Vice-Royauté.

Le quatrième livre des Constitutions traite de façon spécifique «De Gymnasio Ticinensi, et in eo studentium immunitate » (Statuti e Ordinamenti 1925: 157 et suivants). En bref, on refait l'histoire de l'université de Pavie fréquentée, suite à sa renommée, par des étudiants et par des professeurs provenant d'autres pays européens, on y mentionne les avantages obtenus par ceux qui la fréquentent, comme l'exemption des gabelles et des droits pour les maîtres et les écoliers, en énumérant clairement les très graves sanctions pécuniaires auxquelles seraient soumis ceux qui dérogeraient aux ordonnances du Sénat et, notamment, ceux qui iraient étudier ailleurs ou qui obtiendraient leur doctorat dans un autre établissement.

Les Ordonnances (Ordines) promulgués par le Sénat de Milan entendaient endiguer le phénomène notable de la diminution de la population estudiantine. La solution adoptée fut celle de l'obligation, pour les étudiants lombards, de fréquenter exclusivement l'établissement Pavesan, alors qu'on en contrôlait la gestion avec des mesures disciplinaires toujours plus sévères et dissuasives. Les autorités citadines, l'Évêque et le Sénat intervinrent à plusieurs reprises dans la répression des émeutes, on publia des bans et des ordres, on menaça par des avis et des peines toujours plus graves, mais tous les ans on se trouvait dans l'obligation de publier à nouveau ces décrets qui, ponctuellement, nétaient pas appliqués.

Deux facultés formaient l'étude et deux collèges de professeurs suivaient les répétiteurs, utriusque iuris et artium et medicinae; le collège autonome des théologiens supervisait l'enseignement de la théologie. Jusqu'à la moitié du XVIIIe siècle, les deux "portiques ", légal et médical, faisaient partie de l'unique corps universitaire; le professeur principal, titulaire de la première chaire de la faculté, avait le devoir d'assurer le bon fonctionnement de son " portique ", administrait la caisse des petites dépenses, avait des rôles de représentation y compris ceux attribués auparavant au Recteur élu parmi les étudiants ; toute initiative qui ne faisait pas partie des devoirs de 
représentation et d'administration quotidienne devait faire référence au délégué du Sénat.

Les cours se donnaient " dans deux grandes pièces contiguës, séparées par une simple cloison, chacune ayant une vaste cour avec des portiques tout autour et de nombreuses classes en-dessous et au-dessus » (Breventano 1570: 12). L'évêque, chancelier de l'établissement, exerçait des fonctions qui non seulement concernaient l'attribution des grades académiques mais aussi le fonctionnement complexe de l'université : il lui était également confié le contrôle de la bonne exécution des ordres émanant des autorités sénatoriales, destinés à interdire aux étudiants de fréquenter d'autres universités, la recherche de locaux adaptés et de fonds nécessaires pour garantir les leçons, le repérage de logements dignes des écoliers, le respect du calendrier des cours, la rénovation des bâtiments scolaires, l'enquête sur les incidents qui se passaient dans l'établissement et la reconstitution des querelles. En outre, l'évêque veillait sur les professeurs par la publication des « rôles » des lecteurs envoyés à Milan au début de chaque année académique, recueillait des informations sur les candidats pour l'obtention d'une chaire, évaluait les nouvelles nominations, promotions et augmentations de traitements, la fidélité à l'enseignement : dans les premières décennies, à côté du devoir de chancelier, l'évêque de Pavie exerçait même celui de délégué du Sénat de Milan, mais progressivement, suite aux contrastes concernant l'exercice des «prérogatives juridictionnelles » entre la Curie épiscopale et le Sénat, entre la Cour de Madrid et la Curie pontificale, les tâches attribuées à l'évêque furent progressivement redimensionnées.

Donc le Sénat finit par jouer le rôle d'arbitre absolu et d'unique responsable des destins de l'université lombarde, fonctionnant même en l'absence d'un projet spécifique de politique culturelle. Cet état de fait ajouté à celui d'être à distance de l'oeil du souverain, a fait naître un processus de crise au sein de l'institution pavesane : à la moitié du XVIe siècle, il y eut également une première tentative du Sénat visant la réforme de l'université, suivie de celles prévues par la Junte sénatoriale sur la réforme de l'institution. Tout au long de ce siècle, les difficultés internes de l'université vinrent s'ajouter à celles du système scolaire-formatif européen, tandis que des discussions fastidieuses engageaient la réflexion des juristes et de nombreux conflits perdurèrent pendant de nombreuses années témoignant ainsi des fortes 
tensions sociales qui, lors d'une période tourmentée pour le pays, provenaient des prérogatives traditionnelles mises en œuvre pour bannir de la ville l'autonomie et l'indépendance de l'enseignement public.

Une ordonnance aux fins protectionnistes fut émise par Philippe II en 1559 visant tous les sujets espagnols et autorisant ces derniers à séjourner uniquement dans les universités de Bologne (Collège d'Espagne), Rome, Naples et Coimbra. Cette disposition fut appliquée à la Flandre en 1570 sur base d'une ordonnance du Duc d'Albe, représentant du Roi catholique et doté des pleins pouvoirs. La politique restrictive de Philippe II concernait la réalisation progressive d'espaces définis selon des critères confessionnels. Ce fait eut, comme première conséquence, l'interruption du circuit d'échanges universitaires dans les territoires impériaux, précédemment encouragé par la constitution d'une nouvelle élite de service, à la cour de Charles Quint et axé de préférence sur des universités « nationales » telles que celles de Louvain et de Pavie, déjà très fréquentées. Dans l'Empire des Habsbourg, à la moitié du Seizième siècle, la peregrinatio academica selon la ligne directrice Ingolstadt - Dôle - Pavie était l'une de plus actives : barrières législatives, dissociation progressive du comportement des étudiants nobles et populaires, séparation confessionnelle, spécialisation des sièges avec nationalisation des degrés mettront fin à la fréquentation dudit circuit et à celle d'autres aussi. Les privilèges d'exemption qui auparavant avantageaient tous les sujets de l'étude, furent réduits et ensuite annulés au cours d'un siècle seulement. Sous une autre forme, survécurent seulement les immunités fiscales au profit des professeurs comme intégrateurs des émoluments qui leur étaient assignés. Les étudiants perdirent entièrement leurs privilèges, les exemptions fiscales et tous les avantages qui, auparavant, avaient rendu Pavie très attractive comme destination d'étude ; par l'emploi des armes, ceux-ci voulurent construire et défendre la liberté et l'autonomie, en faisant même éclater des rixes et des tumultes dans la ville, coïncidant avec les cérémonies d'ouverture et de clôture de l'année académique, pendant le traditionnel «spupillazione » (baptême) des nouveaux, loctroi du doctorat, les festivités du carnaval ou pendant les traditionnelles fêtes religieuses. Les universitaires se battent les uns les autres, s'empoignent avec les élèves des jésuites et il y a également des conflits entre les étudiants «publics » et les collégiens. 
La création des collèges universitaires a marqué de façon indélébile le destin de Pavie comme ville d'étude. Au quinzième siècle, il y avait déjà eu la fondation d'un internat par le cardinal Branda Castiglioni, institution à laquelle on avait donné le nom de saint Augustin, mais c'est seulement au siècle suivant qu'ont été fondés, parallèlement à d'autres entités laïques déjà expérimentées et fonctionnelles, les deux collèges les plus importants de la ville : en 1561, une première fondation voulue par Charles Borromée, archevêque de Milan qui sétait justement licencié à Pavie en utroque iure le 6 décembre 1559 ; et, quelques années plus tard, en 1567, celle fondée par le pape Pie V Ghislieri.

La création à Pavie de ces deux collèges fut en réponse du besoin de l'Église catholique de contrôler le milieu universitaire et aussi de contribuer à l'éducation d'une élite professionnelle nouvelle, fidèle à l'orthodoxie, ouvertes aux professions libérales et pleinement engagé dans l'administration de l'État. Au cours des décennies précédentes, des écrits à tendance hérétiques et certaines œuvres de Luther et des siens avaient été diffusés dans les communautés académiques. En outre, parmi les étudiants de Pavie, couvait une agitation continuelle et certains désordres, qui avaient déjà fait l'objet de plusieurs avertissements de la part des Ducs eux-mêmes, ne contribuaient pas au sérieux des études. De ce fait, chez le cardinal Borromée prit naissance la volonté de fonder un collège pouvant accueillir des étudiants méritants mais dotés de faibles revenus économiques et également de tenir ces derniers dans un milieu moralement sain, éloignés des mauvaises mœurs répandues. Par la bulle papale du 15 octobre 1561, le pape Pie IV, oncle de Charles Borromée, fonda à Pavie un nouvel internat érigé dans une propriété qui appartenait à sa famille L'architecte Pellegrino Pellegrini initia les travaux peu de temps après mais l'institution accueillit ses premiers pensionnaires en 1581 seulement et la réalisation complète de l'œuvre fut accomplie grâce à l'engagement de Frédéric Borromée, neveu de Charles, lui-même archevêque de Milan du 1595 au 1631.

A la même époque, suite à une décision du pape Pie IV, a été fondé l'autre pensionnat dont la création était apparue incontournable de l'expérience de vie du frère Michel Ghislieri, futur Pie V, qui avait fait ses études de philosophie et de théologie au sein du couvent dominicain de saint Thomas d'Aquin à Pavie. Une fois devenue 
souverain pontife, en l'espace de quelques années, en 1567, Ghislieri mit en œuvre son projet. La bulle officielle fut émise en 1569 mais c'est seulement en 1571 que furent commencés les premiers travaux de construction de l'édifice, sur un plan de l'architecte Pellegrini.

La fondation des internats universitaires à Pavie ne fut pas seulement à l'origine des conflits qui prirent naissance parmi les groupes d'étudiants rivaux, mais, comme il advint également dans d'autres diocèses d'Europe, la création des grands collèges de la Réforme catholique compromit tellement la structure entière du Studio generale e pubblico que les différentes matières didactiques et le privilège de la juridiction personnelle en favorisèrent les pensionnaires, soustrayant ainsi une grande partie de la population estudiantine à l'autorité des Directeurs des études et à celle du Juge d'instance.

A cette période, l'enseignement Pavesan comptait des maitres de grand renom, tout spécialement dans le domaine du droit, comme Francesco Ripa de San Nazzaro, sénateur et comte palatin, lecteur de 1520 à 1535 ; Anton Giovanni Rossi, alexandrin, successivement lecteur à Turin, à Ferrare, à Pise et à Padoue ; Gerolamo Tornielli, appelé par les institutions d'enseignement de Padoue ; Politonio Mezzabarba, sénateur et titulaire d'organismes publics importants ; Francesco Alciato, futur cardinal ; Gaspare Visconti, lui aussi fut nommé cardinal après avoir été évêque de Novare ; et enfin, Aimone Cravetta, déjà professeur à Ferrara et puis lecteur à Pavie de 1556 à 1562. Furent également professeurs de droit, Filippo Decio, professeur à Naples et en France, Francesco Vegio, Giacomo Mandello et Andrea Alciato. Ce dernier, formé à Milan à la culture humaniste, entra, tout jeune encore, à l'université de Pavie où il étudia le droit avec Giason del Maino (Jason Mainus). Il obtint une licence en droit à Bologne et puis il retourna à Milan pour y exercer sa profession. Là il écrivit quelques-unes parmi ses œuvres les plus significatives avec, entre autres, le Adnotaciones au Code justinien. Ensuite, il fut invité à enseigner à Avignon et puis à Bourges où, tout en expliquant le droit romain, il mit en pratique sa culture humaniste. Ses enseignements donnèrent naissance en France à une nouvelle méthode pour les jurisconsultes, qui prévoyait l'acquisition d'une double formation en philologie et en histoire. Alciato fut appelé par la Faculté de droit de Pavie en 1553 où, après une parenthèse dans le domaine de l'enseignement à Bologne, il revint en 1541. L'année suivante, il alla donner 
cours à Ferrare, d'où il partit seulement en 1546 : il resta à Pavie les quatre années suivantes. Plus qu'un réformateur, Alciato fut un authentique innovateur dans l'étude du droit.

Jérôme Cardan conféra aussi un éclat et une renommée européenne à l'enseignement dans la ville au bord du Tessin en tant qu'auteur éclectique de très nombreuses œuvres embrassant les domaines du savoir les plus divers, des mathématiques à la physique, de l'astronomie à la médecine, de la musique à la philosophie.

A côté de ces personnalités à la renommée incontestable, chaque professeur contribua à l'ascension de l'université de Pavie au cours des dernières décennies du Seizième siècle, une ascension qui connut un temps d'arrêt au début, puis une phase de décadence, parallèlement à ce qui se passait au sein d'autres institutions de l'État milanais et, d'une manière plus générale, au sein des universités italiennes qui n'avaient, de toute évidence, pas su ou voulu profiter du souffle de renouveau souvent engendré par celles-ci. Le renouvellement des méthodes qui à l'étranger, et tout particulièrement en France et en Allemagne, avait donné vie à de nouvelles écoles, spécialement dans le domaine juridique, resta sans suite en Italie. Lenseignement Pavesan s'appauvrit rapidement au niveau des enseignants et des élèves : désormais l'époque où les étrangers venaient en grand nombre faire leur doctorat à Pavie touchait à sa fin.

Le Sénat milanais, qui aurait dû faire office de guide et de stimulant pour l'université, vécut un temps de régression, comme attesté par le contenu des décrets promulgués visant une réglementation de la vie de l'université de Pavie. Ceux-ci avaient pour objet le fonctionnement interne et les normes qui régissaient l'institution, l'activité des lecteurs, l'octroi du doctorat, la conduite des étudiants et leur volonté quasi inexistante en matière d'étude, les abus et les désordres qui troublaient la vie de l'université ainsi que les méfaits et les crimes que les étudiants commettaient dans la ville durant des festivités. Pour tout méfait, fut déterminée une sanction, pour tout abus une peine mais la situation se révélait vraisemblablement toujours identique et les décrets toujours plus nombreux ainsi que les peines toujours plus sévères, laissait supposer que la situation empirait.

D’une réalité complexe, on a déjà trace dans le décret du 15 juin 1535 promulgué par l'empereur Charles Quint. Le Sénat, tout en promouvant la nomination d'un nouveau recteur pour l'université des 
«Artistes », était disposé à accepter la reconduction du précédent ou bien de s'en remettre à une personne de confiance dans l'attente de pourvoir à une nouvelle nomination. L'annotation de 1581, rédigée au bas de l'ordonnance, rappelle que, successivement, l'élection du Recteur devint une pratique désuète nonobstant les sévères rappels du Sénat, au point que l'on arriva à nommer un étudiant « faisant fonction " à la place du recteur qui, lors de la présentation de son mémoire de licence, se présentait dans l'amphithéâtre précédé des enseignes, recevait les honneurs comme s'il avait été le véritable Recteur et s'asseyait à la droite du Podestat (Ordines 1743: 15). A la même période sont promulgués deux décrets ordonnant aux lecteurs de ne pas procéder à la dictée des leçons, mais bien d'y apporter des commentaires oraux (Statuti e Ordinamenti, 1925: 152) ${ }^{2}$. Les mesures se succèdent sans résultat apparent. Un ordre écrit du Sénat, en date du 23 juin 1554, émis au nom de l'Empereur Charles Quiny, confirmait que les étudiants pavesans ne pouvaient pas assumer de fonctions universitaires dans leur ville, comme déjà prévu par les Statuts (Ordines 1743: 50). Ledit décret fut le résultat de protestations visant la nomination de Luca Bonamico, comme vice-recteur des Artistes faisant fonction en l'absence du Recteur : comme il s'agissait seulement d'une brève période - affirma le Sénat - il ne sembla pas opportun de le démettre de ses fonctions mais il apparut souhaitable qu'une telle situation ne se reproduise plus dans le futur (Ordines 1743: 51).

La même année, le 7 novembre, au nom de Philippe, roi d'Angleterre et duc de Milan et afin d'éviter d'ultérieures dissensions, il fut interdit aux étudiants déjà en ordre utile d'inscription, de s'inscrire dans un autre groupe, sous peine d'être accusés de production de faux documents. Il faut avoir à l'esprit que les groupes (nationes) étaient composés d'étudiants provenant de la même ville ou région, ayant à leur tête, dans le cas des italiens, le Consiliarus. Les ultramontains, c'est-à-dire les étrangers (exception faite pour les espagnols, assimilés aux pavesans) étaient eux aussi répartis par groupes selon la nationalité de leur responsable auquel l'on avait attribué le titre de Prior. Un décret ultérieur, promulgué le 10 décembre 1670, comme rappelé en annotation de bas de page dans le décret du 23 juin 1555, établit

2 Décrets promulgués le 26 Novembre 1534. 
que «Scholares Papienses, vel Hispani non possunt creare nationem » (Statuti e Ordinamenti 1925: 38-40).

Parallèlement aux discussions qui tenaillaient les milieux politiques et ecclésiastiques, une grande importance fut attribuée, dans les actes publics et privés, au droit de priorité. Les étudiants qui entraient dans l'amphithéâtre épiscopal pour y recevoir leur diplôme de licence ou qui participaient, en même temps que les lecteurs à quelque manifestation publique, étaient précédés par les appariteurs de l'université portant les enseignes, celle des Juristes et celle des Artistes, selon les dispositions du Sénat datées du 16 octobre 1556 (Statuti e Ordinamenti 1925: 158).

Deux ordonnances émises au nom de Philippe, à présent roi d'Espagne et d'Angleterre, outre au dux Mediolanensis, datées respectivement du 28 Mai et du $1^{\text {er }}$ Juin 1556, avaient pour objet les examens de maitrise des recteurs (Ordines 1743: 57-58). Dans la première, le Sénat s'étonnait (mirati sumus vehementer) du cas de Giorgio Cotta, recteur des Artistes qui, comme il en témoigna lui-même, souhaitant être soumis à l'examen de maîtrise pour démontrer ses connaissances, sétait vu objecter par le corps professoral que les recteurs en étaient dispensés, toutefois, moyennant compensation, ils déclaraient être prêts à entendre sa prestation. Une telle démonstration d'avidité parut incroyable aux yeux du Sénat qui demanda à s'assurer de la véracité des faits tout en décidant, en tout cas, que Cotta pouvait présenter son examen de maîtrise gratuitement, en présence de tout le corps professoral, sous peine d'une amende de 10 doublons d'or ou d'autres sanctions encore à discrétion du Sénat lui-même. Les professeurs, taxés d'extorsion, répliquèrent combien il leur paraissait indécent que les recteurs, ayant atteint le doctorat, présentent leur examen de mâ̂trise. Le cas ne fut pas résolu facilement : une deuxième ordonnance, en date du $1^{\text {er }}$ juin 1556, disposa qu'une fois abrogé le privilège qui exemptait les recteurs de leur examen de maîtrise, ces derniers seraient alors tenus ultérieurement de présenter ledit examen sans avoir à payer le moindre honoraire "qui dignitate omnes antecellunt».

Le fait décrit ici assume une valeur exemplaire, il s'agit d'un problème entraînant pour tous une certaine perte : d'une part, pour les recteurs, la perte du privilège d'être exemptés de lexamen et, d'autre part, pour les professeurs, la perte des honoraires qu'ils soutiraient aux recteurs. On comprend parfaitement le déclin du processus de 
nomination des recteurs faute de candidats : la fonction, déjà onéreuse en soi, comportait à présent, comme pour tous les autres, une part d'étude.

On aurait pu s'attendre, de la part du Sénat milanais, à la prise en charge de l'importante question du renouvellement des études mais l'ordonnance du 9 Avril 1573 démontre encore une fois que l'on accordât plus d'importance aux problèmes inhérents à l'université, tels que celui du droit à la priorité lors de manifestations (Ordines 1743: 94-95).

S'il est impossible de parler de censure de la part du gouvernement espagnol concernant l'enseignement, il est légitime d'en signaler le contrôle ou tout au moins l'orientation. Une autre restriction fit jour en date du 9 mai 1591 quand le Sénat imposa aux professeurs (sous peine de sanctions) un type d'enseignement faisant abstraction de tout vent de renouveau touchant, par exemple, le domaine juridique suite aux enseignements d'Alciato (Ordines 1743: 142) : « Professores (...) abstinere debere a cumulatione Doctorum recentiorum ». On mettait donc en évidence l'excessive condescendance des lecteurs vis-à-vis de leurs étudiants, à qui ils dictaient personnellement les leçons, mot à mot, " sed etiam eadem singulis (...) repetere soleant ", favorisant ainsi la paresse des plus négligents et pénalisant les plus diligents. En même temps, le Sénat révélait que les professeurs « vel ambitiose, vel ut sibi ipsis suppellectilem ad praxis comparent ", et ordonnait qu'en matière de cours, on doive s'en tenir aux textes des critiques classiques et s'abstenir de l'ajout de citations plus récentes afin de " ne pas abasourdir les oreilles des étudiants par des notions inutiles »: toute personne qui ne s'en tiendrait pas aux ordonnances, serait condamnée par le Sénat et ferait l'objet de sanctions supplémentaires.

Il n'est pas étonnant dès lors que septante ans plus tard, en 1661, les professeurs de l'université de Pavie, invités à présenter leur programme d'enseignement, aient déclaré de s'en tenir aux « longevae et inveteratae Ticinensis Gymnasii consuetudini » et de suivre « suorum antecessorum ordinem, ab eorum verstigiis non aberrando nec deflectendo ", sans aucun doute en hommage à la réputation des anciens maîtres mais aussi pour indiquer, d'une part, une certaine stagnation dans le domaine de la pensée et de la recherche et, d'autre part, leur adhésion totale au type d'enseignement proposé par les autorités espagnoles (Peroni 1925: 122). 
Dans toutes ces ordonnances, n'apparaît jamais ce qui aurait dû occuper la première place : l'exhortation à un renouveau dans le domaine des études dû à l'évolution et l'action du Sénat apparaît bel et bien comme étant un véritable frein à toute initiative personnelle (Ordines 1743: 142)

Le rapport entre les universités et les organes décisionnels de la monarchie espagnole nétait pas partout homogène. Les sollicitations et les interventions suscitaient l'intérêt du roi et des ministres ainsi que l'ingérence et la réglementation en matière de politique universitaire. Ils le faisaient de façon officielle en procédant à de fréquentes, et parfois minutieuses, inspections, les visitas, déférées à des personnes importantes, choisies souvent de façon avisée parmi les nombreux dignitaires dotés de toutes les qualités requises au niveau des compétences et de la fiabilité. Les visitas stimulèrent la prise de mesures opportunes contre l'esprit de routine et l'inertie professionnelle et dans d'autres cas, celles-ci constituèrent de véritables sources de tensions mettant en lumière certains aspects délicats de la vie politique. Le contrôle du souverain sur les écoles et les universités s'effectuait à travers le "visiteur " qui avait le devoir d'informer directement le souverain (le cas de la réalité castillane est des plus exemplaires : Philippe II fut informé des problèmes existant au sein des écoles de Salamanque par un de ses « visiteurs ", envoyé sur place afin d'établir ce qui se faisait sans en avoir reçu l'ordre, de qui en était la faute et quelles solutions s'avéraient opportunes.

L'interventionnisme espagnol dans la vie académique provenait de la préoccupation de garantir une formation rigoureusement orthodoxe, outre à être efficace, aux futurs «grands commis» de la monarchie ainsi que de la perception du rôle de l'université comme sujet essentiel dans la vie politique, croisement délicat entre les logiques de pouvoir et d'opinion.

En effet, le contrôle ponctuel des formations des théologiens et des juristes était indispensable à une monarchie qui avait fait de la tutelle de l'Église post-concile de Trente et de la fiabilité de ses propres tribunaux, des éléments fondamentaux d'autoreprésentation interne et internationale. Les juristes siégeaient au sein des plus grands organismes de gouvernement de Milan, Naples et du Nouveau Monde.

3 Décret du 9 Mai 1591. 
Outre leur loyauté indéfectible au catholicisme et leur indiscutable compétence professionnelle, le pouvoir royal devait pouvoir compter avant tout sur l'immédiate obéissance de ces derniers. Pour Philippe II, letrados, les juristes et les théologiens devaient être contrôlés au tout début de leur carrière, et intervenir à la racine, dans les universités, pour tenter d'éviter, par des avertissements explicites, les attitudes de dissension présentes dans le corps académique. Dans une lignée telle que celle de la couronne Habsbourg, les risques de subversion nétaient pas liés à la « grandeur des Empires ou à la diversité des nations ", comme l'attestait une opinion répandue parmi les écrivains politiques mais bien aux orientations idéologiques, au pouvoir de la langue (enseignement, prédication, etc.) et aux doctrines. Même si le contrôle de l'institution universitaire apparaît comme étant l'un des piliers centraux de la Monarquía, de l'autre, il ne manque pas de se décliner selon des formes et directions conformes aux territoires assujettis. Dans cette optique, par exemple, est compréhensible le hiatus entre l'attitude de Philippe II à l'égard des universités hispaniques et flamandes, et le choix de s'en remettre à la médiation sénatoriale pour l'université de Pavie.

Nonobstant le scénario qui commençait à prendre forme au cours de la seconde moitié du Cinquecento, les professeurs de valeur ne manquaient pas, parmi lesquels le juriste de Pavie, Jacques Menocchio (Jacobus Menochius), professeur d'Institutions de droit civil, sénateur qui, dans ses écrits, confirmait les droits de l'État contre l'ingérence ecclésiastique. Ensuite, on ne peut oublier d'autres éminents juristes tels qu'Antonio Merenda, Francesco Pecchio et plus tard Polidoro Ripa, Gerolamo Torti et Stefano Garoni. Parmi les maitres de la philosophie, il faut se remémorer le génois barnabite Alexandre Sauli, évêque à Aléria en Corse et aussi à Pavie.

\section{"Séminaire de vertu ॥}

Les guerres et surtout le fléau de la peste, moins violent qu'à Milan mais suffisant pour bouleverser la ville et ses institutions, contribuèrent certainement à déterminer, dans la cité, une situation d'impasse se répercutant sur l'université. Durant le Dix-septième siècle, les difficultés de l'institution académique furent déterminées par un funeste mélange de facteurs internes et externes. 
La crise du recrutement estudiantin frappa lourdement l'université de Pavie, phénomène qui toucha de la même manière toutes les universités de la Péninsule. La création des Studia nationaux, limitant géographiquement le recrutement, provoqua la réduction du flux des étudiants étrangers et réduisit la mobilité des époques précédentes, ayant atteint l'apogée de sa splendeur à l'époque médiévale. Les normes de protection promulguées par les organes gouvernementaux prohibèrent aux jeunes de se rendre hors de l'État pour étudier et de ce fait, à partir de la moitié du Seizième jusqu'aux siècles suivants, les collégiens constituèrent presque la moitié de la population estudiantine à Pavie. Les collèges universitaires assurèrent le maintien de l'enseignement de Pavie à l'intérieur du système composite des institutions éducatives qui étaient sur le point de se former et ce, précisément à l'époque espagnole. Est significatif le nombre de collégiens présents parmi les étudiants : entre 1567 et 1699 entrèrent au Ghislieri 1.103 étudiants, tandis que le Borromeo en accueillit 797 entre les années 1588 et 1699. En supposant, pour chaque élève, une présence quadriennale en internat, la moyenne annuelle des présences d'étudiants au collège, pour tout le Studium, dépassait les soixante unités. Ceux-ci assurèrent la survie de l'université, spécialement dans les moments de crise : des cinq cents étudiants qui peuplaient l'université au commencement du Cinquecento, après un siècle on descendit à septante unités seulement pour les deux Portici (Legisti et Artisti), pour remonter successivement à la centaine dans les dernières décennies du Seicento.

En Lombardie, l'initiative pontificale de concéder la faculté d'accorder le titre doctoral au corps universitaire, collèges et académies, fut poursuivie également par les autorités espagnoles qui, simultanément, introduisirent les qualités de noblesse et de naissance pour l'accès aux collèges citadins des juristes et des physiciens. Les statuts corporatifs de ces collèges, qui admettaient seulement des doctores des familles dont les membres avaient été anoblis depuis quelques générations parmi les cives de Pavie, interdisaient de fait l'entrée aux docteurs étrangers, les entravant ainsi dans l'exercice de leur profession. Le 9 décembre 1637, le Sénat interdisait aux lecteurs de l'université de Pavie l'acceptation d'une charge publique et, par conséquent, imposait l'obligation de se dédier à l'enseignement à plein temps, mettant en danger l'emploi et le salaire (Ordines 1743: 269). Cette 
situation, qui circonscrivait la liberté professionnelle du doctor, du médecin ou de tel ou tel juriste, ajoutée aux maigres disponibilités du Sénat de rétribuer les professeurs par des salaires adéquats et intéressants, contribua, de manière déterminante, à l'exclusion progressive de Pavie du circuit des professeurs « étrangers » et à la réorganisation académique dans le cadre local.

La municipalisation du corps professoral de Pavie fut désormais évidente. Le Sénat, à partir de la fin du Seizième siècle, pour accroître le montant des salaires des professeurs récupéra les privilèges traditionnels et recourut à une formule de rémunération indirecte à travers l'immunité fiscale, dans l'optique de la politique financière générale de l'État milanais. Ce privilège, concrétisé par l'exemption des droits sur les marchandises et biens d'usage personnel ou professionnel, pour les docteurs de l'enseignement, fut étendu à l'entière gamme de taxation, et également à celles, particulièrement appréhendées, sur les logements militaires pour ceux qui possédaient des biens dans le Milanesado. Le corps professoral de l'université de Pavie se présentait désormais, pour la plupart de ses membres, originaires du territoire, avec une réduction marquée de la mobilité, pratique très diffuse à l'époque ducale.

Multiples furent ainsi les causes qui, de la disparition de la cour ducale à la crise économique et aux évènements agités qui bouleversèrent longtemps le territoire, conduisirent à l'accentuation de la dimension provinciale, exprimée également par le corps académique.

En gage de survie de l'enseignement public, continua à persister le lien évident avec le Sénat : protection et conservation de l'université signifiaient aussi protection et conservation des traditions lombardes, du prestige, de l'autonomie, des prérogatives et du pouvoir du Sénat lui-même. Au cours du passage aux dépendances directes de la couronne espagnole, le Sénat et le gouverneur se firent une place dans l'administration de la chose publique. Au Sénat de Milan, résumé des traditions de puissance et de prestige du patriciat lombard, les Constitutions de Charles Quint avaient confié le contrôle et le gouvernement de l'université de Pavie : l'institution, liée à la Magistrature suprême de l'État de Milan depuis des décennies, faisait dorénavant partie intégrante de l'État même.

Le gouverneur, représentant du souverain, chercha à plusieurs reprises de soustraire l'université à l'État, et il ne manqua pas aux 
universitaires les occasions pour se montrer fidèles aux traditions lombardes et au Sénat milanais et de résister aux tentatives de réduction des compétences de celui-ci, en se proclamant contraires à ce genre de «novedades perniciosas que feran la entera perdicion desta Universidad» (ASMi Studi p.a. 392).

Le 4 septembre 1638, le Sénat fit paraître une série d'ordonnances signées par Filippo Meda dans le but de développer l'université, d'accroître le nombre des étudiants et de protéger ceux qui désiraient tirer profit de leurs études (Ordines 1743: 270-271). Les textes reflétaient les avertissements du siècle précédent, répétant l'interdiction pour les «Sujets de ce Domaine» de fréquenter une autre école que celle de Pavie, sous peine de graves sanctions, mettant l'accent sur le fait que les transgresseurs n'auraient pu obtenir leur doctorat à Pavie, ni s'inscrire au Collège des Docteurs (s'ils avaient obtenu ailleurs un diplôme universitaire) et donc de ne pas pouvoir exercer leur profession. Aucune justification nétait acceptée, même pas celle d'avoir dû résider en d'autres villes situées hors de l'État pour l'exercice d'autres fonctions. Pour ceux qui désiraient obtenir le diplôme de maîtrise, l'inscription dans le Livre des matricules était obligatoire. Les appariteurs étaient chargés de la détention dudit Livre et du contrôle des inscriptions annuelles, pratique requise comme témoignage de la continuité dans les études et comme dissuasion aux inscriptions una tantum, sans fréquentation des cours. Les appariteurs devaient signaler au Podestat de Pavie chaque enregistrement effectué dans ce Livre pour que celui-ci note esattissimamente (le plus exactement possible) les présences des étudiants aux cours universitaire. Pour aspirer au diplôme de maîtrise, il fallait avoir la fede, certificat de bonne conduite tenue au cours des années d'étude. Les «spupillazioni » (baptêmes) apparaissaient comme de véritables spoliations au détriment des étudiants et aussi de leurs parents en visite. Ces mêmes actes goliardiques causaient des désordres publiques avec, comme amère conséquence, que les familles les plus en vue de l'État décidèrent souvent d'éloigner leurs enfants de la ville causant le discrédit de l'institution : le Sénat menaçait de prison et d'exclusion de Pavie tous ceux qui auraient été suspectés de ces actes, acceptant également les délations anonymes pour découvrir les coupables, et récompensant les dénonciateurs avec un tiers du montant de l'amende et cela «voulant, sera tenu secret» (Mortier 1958: 525). Vu l'importance de ces ordonnances, le Podestat était tenu de les 
faire afficher dans les lieux habituels et de les réitérer année par année (" et que chaque année celui-ci les fasse à nouveau publier »).

En réalisant sa politique de maintien de l'université, l'attention du Sénat se concentra sur la fonction d'attribution du doctorat. Depuis la fin du XVIe siècle, la licence, la licentia docendi, avait perdu partout sa signification originaire d'habilitation exclusive à l'enseignement ; les titres d'étude délivrés au nom des souverains dans des domaines respectifs acquirent une physionomie professionnelle jusqu'à être reconnus comme réquisit à l'accès aux professions libérales et à toute fonction publique non exécutive. Les universités, en modifiant leur rôle et leur physionomie sociale, se préparaient à devenir l'un des nombreux centres de formation professionnelle et à ne plus être des lieux de recherche privilégiés, de « travail intellectuel et scientifique désintéressé » (Le Goff 1977: 171).

La contradiction apparente fut que, si d'une part le doctorat de Pavie devint indispensable dans l'exercice de la profession juridique, de l'art médical et pour l'acquisition des bénéfices et des dignités ecclésiastiques, d'autre part le Sénat pouvait en dispenser tous ceux qui avaient obtenu ailleurs leur diplôme d'études. La licence de l'université de Pavie conserva quand même sa valence de licentia ubique docendi, mais grâce à la reconnaissance du Sénat, celle-ci acquit un caractère préférentiel. Une telle attribution représenta seulement de manière formelle le point de force du Studium, au point que la concurrence ne vint pas des sièges extérieurs que les sujets, nonobstant les interdictions, continuaient à fréquenter, mais bien d'autres institutions qui, dans l'État, délivraient des licences, comme les Comtes palatins, les collèges professionnels et tous les autres organes d'enseignement.

D'un côté le Sénat persévéra dans la promulgation d'une série d'ordres afin de garantir le sérieux de la licence de Pavie, mais d'un autre côté, les dérogations semblaient devenir la règle dans un système scolaire supérieur dans lequel le rôle central de l'université était en train de perdre toute garantie. Le Collège professionnel des jurisconsultes de Milan démontra être une structure éducative complètement autonome et autosuffisante par rapport à l'université de Pavie en conférant des doctorats dans diverses disciplines.

Le Sénat, à l'égard de la réalité composite qui se façonnait dans la capitale, prit une position qui, tout en étant en harmonie avec le rôle de gardien et de garant des traditions de l'enseignement général et 
public qui lui était attribué par la loi provinciale, ne put tenir compte, après le Concile de Trente, des exigences d'un système éducatif qui, en assumant une nouvelle physionomie, tentait de se faire une place sur le territoire et à se diviser en une pluralité d'institutions concurrentes, publiques et privées, laïques et religieuses. Le Magistrat réaffirma le monopole de Pavie à l'égard des titres d'étude tout en admettant une dérogation pour le Collège milanais, adoptant ainsi une position médiane : le système traditionnel était formellement sauf et la loi provinciale, les ordres du Sénat, le droit en vigueur ainsi que les traditions lombardes étaient respectés.

Le modèle milanais fut étendu et constitua de fait dans le Milanesado (Milanais) un système parallèle à celui traditionnel, centré sur l'université de Pavie, dans lequel la formation culturelle, l'apprentissage professionnel ainsi que l'attribution des titres d'étude faisaient abstraction presque in toto de l'université. Au XVIIe siècle, dans toutes les villes lombardes, furent mis en œuvre des cours (letture) particulièrement encouragés par les collèges professionnels municipaux et tout particulièrement réservés à la formation des futurs professionnels, médecins et juristes citoyens.

Par la situation jusqu'à présent décrite, furent avantagées les écoles privées qui, suite à la décadence du Studium, commencèrent à proliférer au cours de la seconde moitié du Cinquecento. Luniversité de Pavie allait ainsi à l'encontre d'une période de décadence qui favorisa l'exode des étudiants et des professeurs vers Milan, près les Écoles Palatines et le Collège jésuite de Brera. Les Écoles Palatines, nées officiellement à Milan, entre 1601 et 1605, du renouvellement des anciennes écoles du Broletto, disposaient de quatre chaires principales : institutions civiles, rhétorique, mathématique et grec. Un décret du Sénat du 11 juillet 1668 les mettait sur un même pied d'égalité que l'université de Pavie, en y officialisant le cours de droit : celui qui l'aurait fréquenté pendant une année, pouvait réduire à quatre ans le cycle d'études de Pavie.

Les dispositions se succédèrent selon des textes auxquels on n'apporta pas beaucoup de modifications (Statuti e Ordinamenti 1925: 158). Le 12 janvier 1661, la situation à l'université paraissait inchangée car le texte de l'ordonnance du Sénat, ratifié par Carlo Antonio Belcredi, s'inspirait de ceux de 1638 et de 1651, même si présentant quelques nouveaux ajouts (Ordines 1743: 318-319). 
Le problème de la concession de la part de certains privés, en vertu d'antiques privilèges, du titre de docteur, médecin ou notaire fut délibéré par le Sénat le $1^{\text {er }}$ Février 1661 avec la conviction de se trouver face à un sérieux problème. Le décret, ratifié par Francesco Sadarini, «ordonne à tout et chacun Comte Palatin, qui prétend, en vertu de quelconque titre ou privilège, de pouvoir en cet État de Milan instituer des Docteurs, Médecins et Notaires» de présenter, dans le délai d'un mois, au Secrétaire Royal (Regio Segretario) ses titres et privilèges «sous forme authentique», sous peine d'immédiate disgrâce (Ordines 1743: 320).

Ladite résolution ne dût pas être appliquée à court terme car le 22 avril 1667, le Sénat milanais émit un autre décret ratifié par Belcredi sur la transparence des précédents et menaçant les familles des étudiants qui étudiaient hors de Pavie au cas où les amendes imposées n'auraient pas été honorées; on ne manqua pas d'insister que seule l'université pouvait concéder le doctorat et on décida de l'expulsion des professeurs qui auraient permis aux étudiants d'autres institutions de se rendre à Pavie pour la présentation de leur licence (Ordines 1743: 333-335).

La séquence des documents s'enrichit par l'édit du 15 avril 1679, ratifié par Carlo Maria Maggi (Ordines 1743: 390-391 ; Statuti e Ordinamenti 1925: 186-189): les étudiants qui troublaient les leçons seraient chassés de la ville sur dénonciation des lecteurs et des appariteurs; les lecteurs devaient faire arriver à la présentation de la licence seulement les étudiants méritants par degré de culture et moralité, certifier leur fréquentation aux cours, en tenant compte du fait que, en cas de fausses déclarations, ils pouvaient être suspendus de leur charge; les lecteurs étaient autorisés à donner des leçons privées et à procéder à des répétitions selon des horaires différents de ceux de leurs collègues et ceci sans entraver l'enseignement régulier ni les autres matières; on ne manquait pas d'insister sur le comportement des appariteurs envers les professeurs et les écoliers et sur le devoir de la part du podestat et des officiers judiciaires de rendre publique l'ordonnance et de la publier à nouveau chaque année.

Dans la proclamation du 7 avril 1690, le sénateur Giulio Cesare Pagano, podestat de Pavie, rappelait, en onze points, les normes principales «ad rectum regimen Regiae Universitatis», normes qui devaient être par tous «inviolabiter servanda», et particulièrement 
celles à l'égard des contrôles de la présence des étudiants aux leçons et des attestations de fréquentation et de profit délivrées à ceux-ci par les professeurs qui, confiées aux appariteurs généraux, devaient être enregistrées par ces derniers dans un livre spécial, approuvé périodiquement par les professeurs (Statuti e Ordinamenti 1925: 188-189). Dans un cas seulement le Sénat dispensa un lecteur de sa charge d'enseignant, Giovanni Antonio Pulzio: celui-ci, durant la période au cours de laquelle il exerçait la charge de juge près le tribunal de Crémone, ne pouvait bénéficier ni du salaire ni de l'immunité concédés aux lecteurs (Statuti e Ordinamenti 1925: 160)4.

En ce qui concerne le Dix-septième siècle, il faut relater le bref, mais important enseignement de Gaspare Aselli (Gasparus Asellius), professeur d'anatomie originaire de Crémone et découvreur des vases chylifères. Des noms importants ne manquèrent pas dans les domaines des études philosophiques, théologiques et mathématiques surtout parmi les servites : Enrico Antonio Borgo, plus tard professeur à Pise et général de l'ordre; Filippo Ferrari, mathématicien, géographe, topographe et puis général; Giovan Battista Drusiani, mathématicien, astronome et cosmographe; Gerolamo Puricelli, lui aussi général; Francesco Pertusati, moine olivétain et évêque de Pavie; Giovanni Battista Colombini, nommé général des frères mineurs. Et encore Bartolomeo Serravezza; Placido Tito; vers la fin du siècle (1699) fut appelé le mathématicien jésuite Gerolamo Saccheri, maître de sciences physiques et naturelles qui, à Pavie, occupa la chaire de mathématique pendant plus de trente ans, jusqu'à sa mort commentateur d'Euclide et précurseur des géométries non euclidiennes; l'augustinien Filippo Lachini, lecteur de logique, de philosophie et créateur de la première bibliothèque publique de Pavie.

Nonobstant les difficultés internes et les pressions externes, tout au long du Seicento, ne manquèrent pas les noms importants dans le domaine des études philosophiques, théologiques et mathématiques. La pénible évolution de l'université de Pavie entre $\mathrm{XVI}^{\mathrm{e}}$ et $\mathrm{XVII}{ }^{\mathrm{e}}$ siècle ne fut cependant pas pleinement perçue en dehors de l'État. L'impression qu'on en retirait de l'extérieur nétait pas des plus satisfaisantes. Il suffit de penser à la substantielle méfiance manifestée en 1696 par Juan Francisco Pacecho Téllez-Girón, duc de Uzeda et vice-roi de

4 Ordonnance du 8 Janvier 1693. 
Sicile qui estimait le Studium «du Duc de Sfortia», de même que la Sapienza romaine et l'école de Salerne, comme étant des institutions assez désinvoltes, décernant des licences à des étudiants qui n'avaient pas fréquenté régulièrement les cours académiques, se révélant être de véritables fabriques de titres de docteur, outre à être des dispensatrices de faux titres. En considération de cela, le vice-roi de Sicile ordonna que soit interdit aux licenciés en médecine et in utroque iure auprès de telles institutions et auprès d'autres universités étrangères « d'exercer les Offices de Docteur en Droit, Médecine, Physique et Chirurgie » (Cesino e Foglietta 1700: 270). Et le Sénat de Palerme demanda au souverain d'autoriser la fondation d'une université comme advenu en Lombardie : « Dans l'Etat de Milan, à Pavie, on étudie et on est proclamé docteur » (Archivio Comunale di Palermo).

L'image que les autorités espagnoles et celles de l'île voulaient transmettre des institutions scolaires supérieures en Sicile était positive et pour ce faire, celles-ci n'hésitaient pas à faire apparaître sous un jour défavorable les autres universités de la Péninsule, n'excluant même pas celle de l'Estado de Milan. Plus grande ingérence du gouvernement central ou seulement une politique à rayonnement local limité ?

$$
* * *
$$

A la lumière des témoignages recueillis au cours des diverses enquêtes réalisées au Dix-huitième siècle, dans le cadre de la réforme thérèsienne des études en Lombardie, la cause principale de la décadence de l'enseignement public sera indiquée, dans la perspective de la construction d'un nouveau modèle d'université, dans les "privilèges de conférer les licences $[\ldots]$ ceux-ci étant déjà communs aux comtes palatins, aux collèges publics et privés et aux ordres religieux [...] tandis que la licence au contraire, ne devrait pas être octroyée si le cours régulier des études dans cette même Université nétait pas arrivé à terme» (ASMi, Studi p.a. 375$)^{5}$.

Au cours des décennies à cheval entre le XVIe et le XVIIe siècle, la compacité du système éducatif, qui considérait l'enseignement général et public de Pavie comme le centre propulseur de la vie culturelle et de la formation professionnelle dans l'État entier, se fissure:

5 Michele Daverio, Idea di un piano di riforma degli studi ecclesiastici proposto al conte di Firmian, Milano 9 maggio 1767, p. 112-116. 
à Pavie, siège universitaire, outre aux salles d'étude «sont peuplées [...] les écoles des Barnabites, du Séminaire de l'Évêché, et plus particulièrement celles des Jésuites, dont la résidence se trouve en face de l'Université, rappellent la fréquence des écoliers d'antan, les Écoles publiques quant à elles sont presque abandonnées et sont [et aussi] désertées, la plupart des Collégiens, au lieu de se rendre à l'Université, fréquentent les Écoles qui ont pris place au sein des Collèges, ou encore les Couvents des Ordres Réguliers» (ASMi Studi p.a. $375^{6}$ ).

Ce système fut le résultat du contraste existant entre un projet ambitieux et une réalité complexe et différente des d'institutions vieilles comme l'université ou les écoles magistrales, et nouvelles comme les écoles des ordres tridentins (dans le Milanesado, les Jésuites, les Barnabites et les Somasques jouèrent un rôle principal) tout comme les collèges professionnels - et des coutumes invétérées, comme la formation acquise en privé. Mais sa bonne fortune fut également débitrice d'un nouveau rapport existant entre l'individu et l'institution, si bien que l'individu ne cherchait pas une institution pouvant prendre en charge son parcours d'études, mais bien la réalisation d'un curriculum conciliant les exigences et les projets personnels aux institutions disponibles.

Une fois disparue la dynastie locale et déchue l'incidence d'envergure européenne de l'université de Pavie, exclue également du cercle des pèlerinages académiques des étudiants étrangers et du circuit des centres culturels connus au-delà des Alpes, l'université exerce désormais ses fonctions à l'intérieur d'un système éducatif lombard composite.

\section{Bibliographie}

Almum Studium Papiense. (2013). Storia dell'Università di Pavia. Volume 1: Dalle origini all'età spagnola, Tomo II: L'età spagnola, éd. D. Mantovani, Milano: Cisalpino.

Archivio Comunale di Palermo. (7 Janvier 1697), Consulte del Senato, 1696-1700. FF. 58r-62v.

Archivio di Stato di Milano (ASMi). Studi p.a. 375: lettre de Francesco Sartirana, professeur de l'université de Pavie à Charles-Joseph Firmian,

6 Lettre de Francesco Sartirana, professeur de l'université de Pavie à CharlesJoseph Firmian, ministre plénipotentiaire et gouverneur général de la Lombardie autrichienne, en novembre 1767. 
ministre plénipotentiaire et gouverneur général de la Lombardie autrichienne, en novembre 1767.

Archivio di Stato di Milano, Studi p.a. 375: Michele Daverio, Idea di un piano di riforma degli studi ecclesiastici proposto al conte di Firmian, Milano 9 maggio 1767, p. 112-116.

Archivio di Stato di Milano, Studi p.a. 392: lettre de Madrid du 20 Septembre 1647 au gouverneur Bernardino Fernández de Velasco y Tovar, Condestable de Castilla, duque de Frias ; Archivo General de Simancas, Secretarias Provinciales, leg. 1797, doc. 277 (ca. 1599).

Breventano S. (1570). Istoria della antichità, nobiltà, e delle cose notabili della città di Pavia. Pavia: Appresso Hieronimo Bartholin elle Case di S. Pietro in Ciel Aureo.

Cesino I. e Foglietta. (1700). Pragmaticarum regni Siciliae t. III. Panormi: apud Joseph Gramignani typographum regii palatii.

Le Goff J. (1977). Le università e i poteri pubblici, in Tempo della Chiesa e tempo del mercante. Torino: Einaudi.

Mantovani D. (éd.). (2013). Almum Studium Papiense. Storia dell'Università di Pavia. Volume 1: Dalle origini all'età spagnola, Tomo II: L'età spagnola. Milano: Cisalpino.

Memorie e documenti per la storia dell'Università di Pavia e degli uomini più illustri che vi insegnarono. II. Documenti. (1877-78). Pavia: Tip. Bizzoni.

Mortier R. (éd.). (1958). Dictionnaire encyclopédique Quillet. I. Paris: Quillet.

Ordines Excellentissimi Senatus Mediolani ab Anno 1460 ad Annum 1639. (1743). Mediolani: in Curia regia, semptibus Joseph Richini Malatestae regii ducalis typographi.

Peroni B. (1925). La riforma dell'Università di Pavia nel Settecento, in Contributi alla storia dell'Università di Pavia. Pavia: Tip. Cooperativa.

Statuti e Ordinamenti della Università di Pavia dall'anno 1361 all'anno 1859 raccolti e pubblicati nell'XI centenario dell'Ateneo. (1925). Lettre du 18 Septembre 1516. Pavia: Tip. Cooperativa.

\title{
ADRESSE POUR CORRESPONDANCE
}

\author{
Simona Negruzzo \\ Università di Bologna \\ Dipartimento di Storia Culture Civiltà \\ e-mail: simona.negruzzo@unibo.it
}

